\title{
Concentration changes of organochlorine compounds and polybromodiphenyl ethers during metamorphosis of aquatic insects
}

\author{
MIREIA BARTRONS $\dagger * *$ JOAN O. GRIMALT $\$$ AND JORDI CATALAN $\dagger$ \\ $\dagger$ Limnology Unit (CSIC-UB). Centre for Advanced Studies of Blanes (CEAB-CSIC). \\ Accés Cala St. Francesc, 14. 17300 - Blanes, Catalonia, Spain.
}

$\$$ Department of Environmental Chemistry (IIQAB-CSIC). Jordi Girona, 18. 08034 Barcelona, Catalonia, Spain

* To whom correspondence should be addressed (phone: 3497233 61 01, Fax: 34972

3378 06, e-mail: mbartrons @ ceab.csic.es) 
Table SI1: Data on individual compounds concentration in $\mathrm{ng} \mathrm{g}^{-1}$ dry weight.

\begin{tabular}{|c|c|c|c|c|c|c|c|c|}
\hline \multirow[b]{2}{*}{$\left(n g^{-1} d w\right)$} & \multicolumn{2}{|c|}{ Ceratopogonidae } & \multicolumn{2}{|c|}{ Limnephilidae } & \multicolumn{2}{|c|}{ Chironomidae } & \multicolumn{2}{|c|}{ Polycentropodidae } \\
\hline & Larva & Pupa & Larva & Pupa & Larva & Pupa & Larva & Pupa \\
\hline$\alpha-\mathrm{HCH}$ & 0.12 & $<$ d.l. & 0.01 & 0.11 & 0.04 & $<$ d.l. & $<$ d.l. & $<$ d.l. \\
\hline HCB & 6.01 & 8.26 & 0.09 & $<$ d.l. & 1.18 & 13.57 & 3.11 & 3.20 \\
\hline$\beta-\mathrm{HCH}$ & $<$ d.l. & $<$ d.l. & $<$ d.l. & $<$ d.l. & $<$ d.l. & $<$ d.l. & $<$ d.l. & $<$ d.l. \\
\hline$\gamma-\mathrm{HCH}$ & 7.84 & 12.31 & 0.22 & 1.17 & 6.41 & 25.88 & 0.48 & 1.41 \\
\hline$\delta$-HCH & 0.10 & 0.59 & 0.01 & 0.12 & 0.23 & 2.82 & 0.27 & 0.13 \\
\hline 4,4'-DDE & 2.51 & 3.24 & 1.04 & 2.51 & 3.99 & 14.47 & 4.85 & 5.90 \\
\hline 4,4'-DDT & 0.13 & $<$ d.l. & 0.06 & 1.96 & 0.49 & 0.26 & 1.13 & 0.62 \\
\hline PCB-28 & $<$ d.1. & $<$ d.l. & 0.02 & 0.11 & 0.08 & $<$ d.l. & 0.38 & $<$ d.l. \\
\hline PCB-52 & $<$ d.l. & $<$ d.l. & 0.03 & 0.71 & $<$ d.l. & $<$ d.l. & $<$ d.l. & $<$ d.l. \\
\hline PCB-101 & 1.61 & 3.46 & 0.15 & 0.68 & 0.51 & 9.39 & 1.37 & 0.87 \\
\hline PCB-118 & 1.44 & 3.12 & 0.14 & 0.67 & 0.40 & 2.80 & 1.35 & 1.98 \\
\hline PCB-153 & 2.16 & 3.73 & 0.32 & 1.72 & 1.09 & 4.11 & 2.89 & 5.40 \\
\hline РCB-138 & 1.58 & 3.21 & 0.30 & 1.43 & 0.94 & 4.25 & 1.36 & 2.82 \\
\hline PCB-180 & 0.71 & 1.31 & 0.11 & 0.58 & 0.53 & 1.57 & 1.58 & 3.11 \\
\hline РCB-194 & 0.15 & 0.25 & 0.02 & 0.12 & 0.14 & 0.46 & 0.33 & 0.73 \\
\hline PCB-206 & 1.50 & 1.23 & 0.00 & $<$ d.1. & $<$ d.l. & 0.51 & 0.15 & 0.26 \\
\hline BDE-17 & $<$ d.1. & $<$ d.l. & $<$ d.1. & $<$ d.l. & $<$ d.l. & $<$ d.l. & $<$ d.l. & $<$ d.l. \\
\hline BDE-28 & $<$ d.l. & $<$ d.l. & $<$ d.l. & $<\mathrm{d} .1$. & $<$ d.l. & $<\mathrm{d} .1$. & $<$ d.l. & $<$ d.l. \\
\hline BDE-71 & 0.18 & $<$ d.l. & 0.02 & 0.24 & < d.l. & 0.16 & 0.43 & $<$ d.l. \\
\hline BDE-47 & 0.83 & 2.61 & 0.24 & 1.92 & $<$ d.l. & 2.42 & 3.04 & 4.31 \\
\hline BDE-66 & $<$ d.l. & $<$ d.l. & $<$ d.l. & $<\mathrm{d} .1$. & $<$ d.l. & $<\mathrm{d} .1$ & $<$ d.l. & $<$ d.l. \\
\hline BDE-100 & 0.12 & 0.47 & 0.06 & 0.55 & $<$ d.l. & 0.64 & 0.44 & 1.64 \\
\hline BDE-99 & 0.53 & 1.79 & 0.13 & 1.17 & $<$ d.l. & 0.61 & 1.79 & 3.60 \\
\hline BDE-85 & $<$ d.l. & $<$ d.l. & $<$ d.l. & 0.64 & $<$ d.l. & $<\mathrm{d} .1$ & $<$ d.l. & 1.45 \\
\hline BDE-154 & $<$ d.l. & $<$ d.l. & 0.02 & 0.95 & $<$ d.l. & $<$ d.l. & 1.47 & 2.50 \\
\hline BDE-153 & 0.02 & 0.23 & 0.06 & 0.33 & $<$ d.l. & $<$ d.l. & 1.23 & 3.12 \\
\hline BDE-138 & $<$ d.l. & $<$ d.l. & 0.02 & 0.58 & $<$ d.l. & $<$ d.l. & $<$ d.l. & 1.79 \\
\hline BDE-183 & $<$ d.1. & $<$ d.l. & 0.02 & 0.45 & $<$ d.l. & 0.08 & 1.12 & 2.35 \\
\hline BDE-190 & $<$ d.l. & 0.07 & 0.04 & 0.56 & $<$ d.l. & $<$ d.l. & 0.79 & 1.69 \\
\hline BDE-209 & $<$ d.1. & $<$ d.l. & 0.04 & 1.93 & $<$ d.l. & $<$ d.l. & 2.76 & 4.93 \\
\hline
\end{tabular}


Table SI2: Organochlorine compounds loadings on the main axes of a Principal Component Analyses. First axis explains most of the variance and most of the compounds have a positive loading on it, indicating a general concentration effect. This axis discriminates between larvae and pupae for each group (see figure S1).

\begin{tabular}{lccc}
\hline OCs & Comp. 1 & Comp. 2 & Comp. 3 \\
\hline$\alpha$-HCH & 0.00 & -0.01 & 0.00 \\
HCB & 0.41 & 0.09 & 0.58 \\
$\gamma$-HCH & 0.79 & -0.44 & -0.13 \\
$\delta$-HCH & 0.08 & 0.05 & -0.11 \\
$4,4^{\prime}-\mathrm{DDE}$ & 0.33 & 0.67 & -0.55 \\
4,4 '-DDT & -0.02 & 0.09 & -0.11 \\
PCB-28 & 0.00 & 0.02 & -0.01 \\
PCB-52 & -0.01 & 0.00 & -0.03 \\
PCB-101 & 0.27 & 0.11 & -0.09 \\
PCB-118 & 0.07 & 0.10 & 0.31 \\
PCB-153 & 0.07 & 0.44 & 0.38 \\
PCB-138 & 0.10 & 0.17 & 0.19 \\
PCB-180 & 0.02 & 0.30 & 0.16 \\
PCB-194 & 0.01 & 0.07 & 0.03 \\
Variance explained & $\mathbf{0 . 9 0}$ & $\mathbf{0 . 0 5}$ & $\mathbf{0 . 0 3}$ \\
Cumulative variance explained & $\mathbf{0 . 9 0}$ & $\mathbf{0 . 9 6}$ & $\mathbf{0 . 9 9}$ \\
\hline
\end{tabular}

Figure SI1. Comparison of the scores in the main PCA axis between larvae and pupae of each taxon.

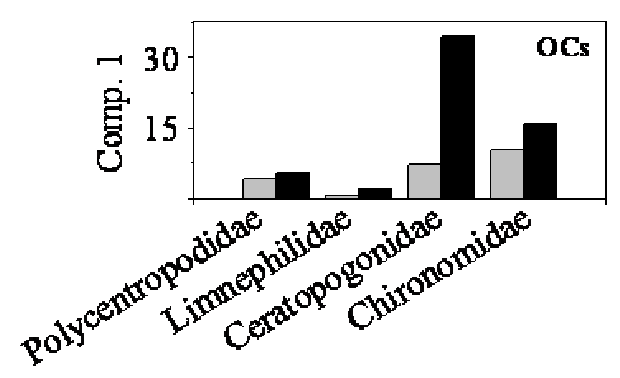


Table SI3: Polybromodiphenyl ethers loadings on the main axes of a Principal Component Analyses. First axis explains most of the variance and all compounds have a positive loading on it, indicating a general concentration effect. This axis discriminates between larvae and pupae for each group (see figure S2).

\begin{tabular}{lccc}
\hline PBDEs & Comp. 1 & Comp. 2 & Comp. 3 \\
\hline BDE-71 & 0.01 & -0.01 & 0.29 \\
BDE-47 & 0.41 & -0.77 & 0.19 \\
BDE-100 & 0.15 & -0.11 & -0.29 \\
BDE-99 & 0.36 & -0.30 & -0.29 \\
BDE-85 & 0.14 & 0.14 & -0.41 \\
BDE-154 & 0.29 & 0.20 & 0.24 \\
BDE-153 & 0.33 & 0.14 & -0.22 \\
BDE-138 & 0.18 & 0.16 & -0.53 \\
BDE-183 & 0.26 & 0.14 & 0.01 \\
BDE-190 & 0.19 & 0.12 & 0.00 \\
BDE-209 & 0.57 & 0.40 & 0.39 \\
Variance explained & $\mathbf{0 . 8 9}$ & $\mathbf{0 . 0 7}$ & $\mathbf{0 . 0 2}$ \\
Cumulative variance explained & $\mathbf{0 . 8 9}$ & $\mathbf{0 . 9 6}$ & $\mathbf{0 . 9 8}$ \\
\hline
\end{tabular}

Figure SI2. Comparison of the scores in the main PCA axis between larvae and pupae of each taxon.

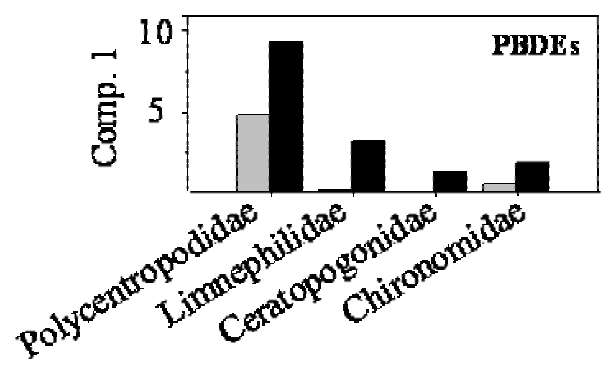

